Technical Note

\title{
Onset of surface tension driven convection in a fluid layer overlying a layer of an anisotropic porous medium
}

\author{
I.S. Shivakumara ${ }^{a, b, *}$, Jinho Lee ${ }^{a, *}$, Krishna B. Chavaraddi ${ }^{c}$ \\ ${ }^{a}$ School of Mechanical Engineering, Yonsei University, Seoul 120-749, South Korea \\ ${ }^{\mathrm{b}}$ UGC-CAS in Fluid Mechanics, Department of Mathematics, Bangalore University, Bangalore 560001, India \\ ${ }^{\mathrm{c}}$ Department of Mathematics, Government First Grade College, Yellapur (UK) 581 359, India
}

\section{A R T I C L E I N F O}

\section{Article history:}

Received 28 July 2010

Received in revised form 10 October 2010

Accepted 17 October 2010

Available online 29 November 2010

\section{Keywords:}

Marangoni convection

Composite layers

Anisotropic porous layer

Beavers-Joseph condition

\begin{abstract}
A B S T R A C T
The paper deals with the criterion for the onset of surface tension-driven convection in the presence of temperature gradients in a two-layer system comprising a fluid saturated anisotropic porous layer over which lies a layer of fluid. The lower rigid surface is assumed to be insulated to temperature perturbations, while at the upper non-deformable free surface a general thermal condition is invoked. Both the Beavers-Joseph and the Jones conditions have been used at the interface to know their preference and prominence in the study of the problem. The resulting eigenvalue problem is solved exactly and also by regular perturbation technique when both the boundaries are insulating to temperature perturbations. It is found that the depth of the relative layers, mechanical and thermal anisotropy parameters have a profound effect on the stability of the system. Decreasing the mechanical anisotropy parameter and increasing the thermal anisotropy parameter leads to stabilization of the system. Besides, the possibility of control of Marangoni convection by suitable choice of physical parameters is discussed in detail.
\end{abstract}

(C) 2010 Elsevier Ltd. All rights reserved.

\section{Introduction}

Convective instability in a fluid/porous layer may occur either due to buoyancy forces or surface tension variations in the presence of temperature and/or concentration, gradients or combined buoyancy and surface tension forces. Each of these convective instability problems in a fluid layer has been studied extensively in recent decades due to their promising applications in engineering and technology. Its counter part in a fluid saturated porous medium has also been investigated extensively but majority of the investigations pertain to buoyancy-driven flows. The developments in the field are amply documented in the literature [1-3]. Convective instability in a liquid saturated porous layer due to surface tension and/or buoyancy forces has also been investigated but it is still in much-to-be desired state [4-6] and references therein.

However, rapid developments in modern technologies during the recent past have posed challenges in studying convective instability problems in more complicated two and multilayer fluid dynamical systems. The use of such composite systems can be found, for example, in the following applications: manufacture of composite materials used in aircraft structures and automobile

\footnotetext{
* Corresponding author at: UGC-CAS in Fluid Mechanics, Department of Mathematics, Bangalore University, Bangalore 560001, India.

E-mail addresses: shivakumarais@gmail.com (I.S. Shivakumara), jinholee@ yonsei.ac.kr (J. Lee).
}

industries, geophysics, bioconvection, nuclear reactors, solid-matrix heat exchangers, crystal growth, directional solidification of alloys and electronics cooling. There exist several investigations pertaining to convective instability in a two-layer system composed of a fluid saturated porous layer over which lies a fluid layer and also different systems of superposed porous and fluid layers.

Nield [7] has investigated the linear stability problem of superposed fluid and porous layers with buoyancy and surface tension effects at the deformable upper free surface by using the Beavers-Joseph slip condition at the interface. The thermal stability for different systems of superposed porous and fluid regions has also been analyzed by Taslim and Narusawa [8]. Chen [9] has implemented a linear stability analysis to investigate the effect of throughflow on the onset of thermal convection in a fluid layer overlying a porous layer with an idea of understanding the control of convective instability by the adjustment of throughflow. McKay [10] has considered the onset of buoyancy-driven convection in superposed reacting fluid and porous layers. Straughan $[11,12]$ has studied a fundamental model for convection in a porous-fluid layer system developed originally by Nield [7]. He has obtained the eigenvalues and eigenfunctions numerically by utilizing the Chebyshev tau method. In particular, the effect of surface tension is also allowed for in the former paper, while in the latter paper, the effect of variation of properties of relevant fluid and porous material on the control of convection is discussed by considering the upper surface to be fixed or stress free. Nield [13] has argued 


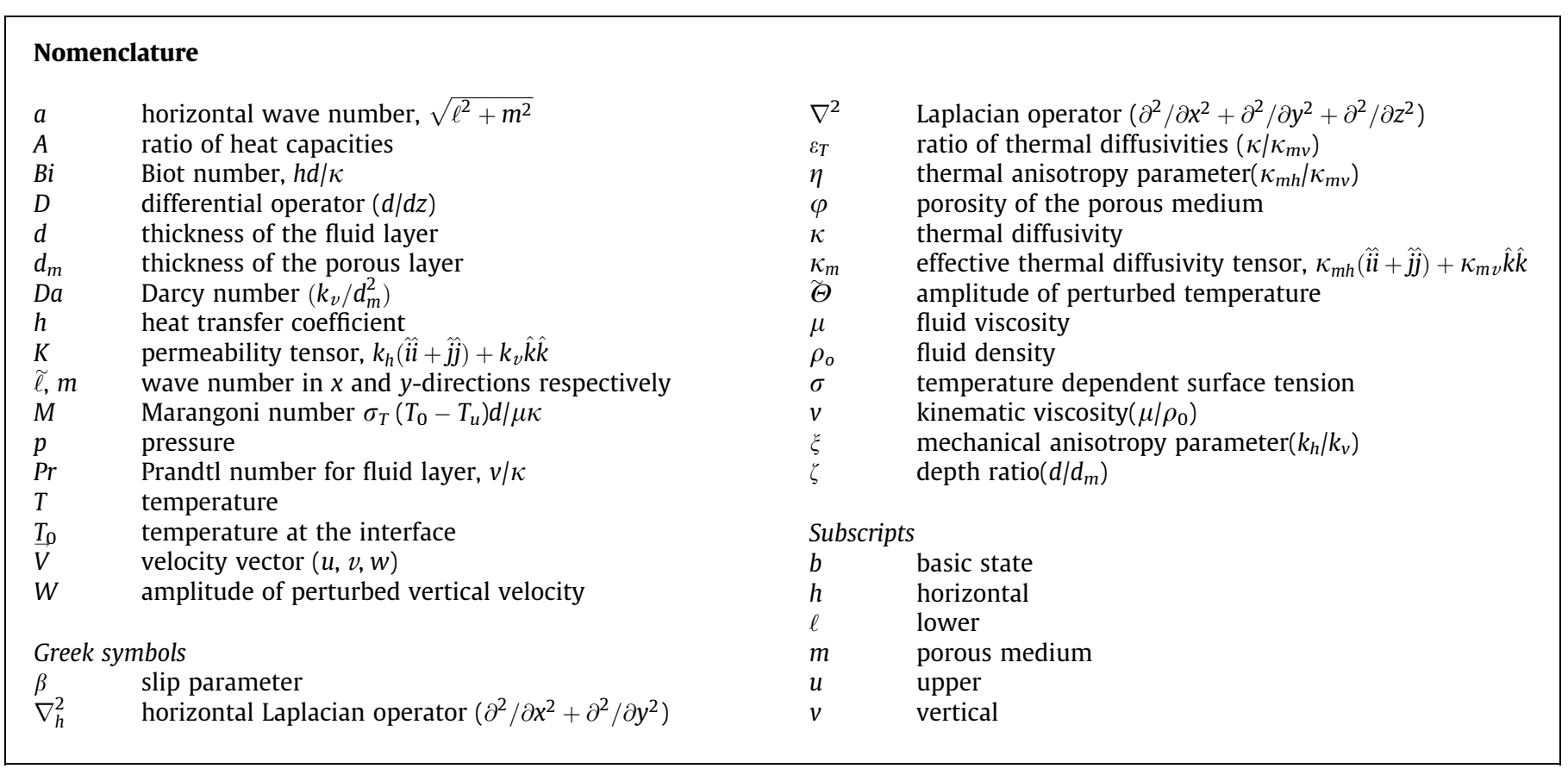

about the modeling of Marangoni convection in a fluid saturated porous medium and has suggested the consideration of composite porous-fluid layer system in analyzing the problem. Khalili et al. [14] have obtained the closed form solution for Chen's model by considering the upper and lower boundaries are insulating to temperature perturbations. Carr [15] has studied penetrative convection via internal heating in a two-layer system in which a layer of fluid overlies and saturates a porous medium. Shivakumara et al. [16] have discussed the onset of Marangoni convection in a composite fluid and porous layers, while Shivakumara and Chavaraddi [17] have investigated the problem by considering the deformation of the upper free surface. Recently, Alloui and Vasseur [18] have reported an analytical study of the stability and natural convection in a system consisting of a horizontal fluid layer over a layer of saturated porous medium.

In all the above superposed fluid and porous layers problems, the porous medium is considered to be isotropic. Nevertheless, in many practical situations the porous materials are anisotropic in their mechanical and thermal properties. Anisotropy is generally a consequence of preferential orientation or asymmetric geometry of porous matrix or fibers and is in fact encountered in numerous systems in industry and nature. Anisotropy can also be a characteristic of artificial porous materials like pelletting used in chemical engineering process and fiber material used in insulating purposes. During the solidification of alloys a dendritic region known as mushy zone separating the melt from the solid forms and this region is regarded as a porous medium in which the permeability and possibly the thermal conductivity may be anisotropic [19]. Castinel and Combarnous [20] were the first to study both experimentally and theoretically the onset of convection in a layer of porous medium with anisotropic permeability.

The onset of buoyancy-driven convection due to heating from below in a system consisting of a fluid layer overlying a porous layer with anisotropic permeability and thermal diffusivity has been discussed by Chen et al. [21]. A review covering the major developments in the field can be found in the book by Nield and Bejan [3]. Nonetheless, Marangoni convection in superposed fluid and mushy layer of mixed solid and liquid phases has become increasingly important in crystal growth, solidification of molten alloys, and other related areas with the advancement of space experimentation under microgravity conditions. For instance there are significant differences in the compositional homogeneity and structural perfection between space grown and ground grown crystals. The unfavorable buoyancy driven convection, sedimentation, and hydrostatic pressure in the process of crystal growth can be suppressed only under reduced gravity environment and as a consequence high quality crystals can be obtained. In view of this, it is imperative to understand control (suppress or augment) of Marangoni convection in the absence of buoyancy driven convection in such systems.

The intent of the present paper is, therefore, to study convective instability in a composite system solely due to temperature dependent surface tension effects at the upper free surface of a fluid layer overlying an anisotropic porous layer. The effect of both mechanical and thermal anisotropy is considered by simplifying it to a horizontally isotropic case. Such a model is of physical relevance to practical situations since many porous structures display anisotropy where the permeability and in turn the thermal conductivity in the vertical direction is different to that in the horizontal plane. At the interface of the porous and fluid media both the BeaversJoseph [22] and the Jones [23] slip conditions are used and a modified Darcy equation is employed to describe the flow regime in the anisotropic porous medium. The lower rigid boundary is insulated to temperature perturbations, while a general thermal boundary condition is invoked at the upper non-deformable free boundary. The resulting eigenvalue problem is solved exactly and an analytic expression for the Marangoni number is obtained and the results are exhibited graphically to assess the effects of the depth ratio $\zeta$, mechanical anisotropy parameter $\xi$, thermal anisotropy parameter $\eta$, Biot number $B i$ and the Darcy number $D a$ along with other physical parameters. When both the boundaries are insulated to temperature perturbations, an analytic expression for the critical Marangoni number is also obtained using a regular perturbation technique with wave number $a$ as a perturbation parameter. It is observed that the results obtained from the regular perturbation technique are in excellent agreement with those obtained from the exact analysis and also the existing results in the literature are obtained as particular cases from the present study. 


\section{Formulation of the problem}

We consider a superposed horizontal anisotropic porous layer of thickness $d_{m}$ underlying a fluid layer of thickness $d$ with no lateral boundaries as shown in Fig. 1. The lower boundary of the anisotropic porous layer is taken to be rigid, while the upper free boundary of the fluid layer at which the surface tension acts is assumed to be non-deformable since for most liquids the capillary number is very small, commonly ranging from $10^{-6}$ to $10^{-2}$. The surface tension $\sigma$ is assumed to vary linearly with temperature in the form $\sigma=\sigma_{0}-\sigma_{T}\left(T-T_{0}\right)$, where $\sigma_{0}$ is the unperturbed value and $-\sigma_{T}$ is the rate of change of surface tension with temperature. The temperature of the lower and upper boundaries is taken to be uniform and equal to $T_{l}$ and $T_{u}$ respectively with $T_{l}>T_{u}$. A Cartesian coordinate system $(x, y, z)$ is chosen such that the origin is at the interface between the fluid layer and the anisotropic porous layer and the $z$-axis is vertically upward.

The governing equations in the fluid and porous layers are:

\section{Fluid layer}

$$
\nabla \cdot \vec{V}=0
$$

$\rho_{0}\left[\frac{\partial \vec{V}}{\partial t}+(\vec{V} \cdot \nabla) \vec{V}\right]=-\nabla p+\mu \nabla^{2} \vec{V}$

$\frac{\partial T}{\partial t}+(\vec{V} \cdot \nabla) T=\kappa \nabla^{2} T$

Porous layer

$\nabla_{m} \cdot \vec{V}_{m}=0$

$\frac{\rho_{0}}{\phi} \frac{\partial \vec{V}_{m}}{\partial t}=-\nabla_{m} p_{m}-\underset{\sim}{\mu K^{-1}} \cdot \vec{V}_{m}$

$\left.A \frac{\partial T_{m}}{\partial t}+\left(\vec{V}_{m} \cdot \nabla_{m}\right) T_{m}=\nabla_{m} \cdot \underset{\sim}{\kappa_{m}} \cdot \nabla_{m} T_{m}\right)$

Here, $\vec{V}$ is the velocity vector, $p$ the pressure, $\mathrm{T}$ the temperature, $\mu$ the fluid viscosity, $\kappa$ the thermal diffusivity, $\varphi$ the porosity of the porous medium, $A$ the ratio of heat capacities and the subscript $m$ denotes the porous medium. The permeability and thermal diffusivity tensors of the porous medium are assumed to be constant and to have principal axes aligned with the co-ordinate system so that $K^{-1}=K_{x}^{-1} \hat{i} \hat{i}+k_{y}^{-1} \hat{j} \hat{j}+k_{z}^{-1} \hat{k} \hat{k}$ and $\kappa_{m}=\kappa_{m x} \hat{i} \hat{i}+\kappa_{m y} \hat{j} \hat{j}+\kappa_{m z} \hat{k} \hat{k}$. We restrict to horizontal isotropic porous media and consider $k_{x}=k_{y}$ $\left(=k_{h}\right)$ and $\kappa_{m x}=\kappa_{m y}\left(=\kappa_{m h}\right)$. It may be noted that the permeability and effective thermal diffusivity in the horizontal and vertical directions in an anisotropic porous layer are denoted by $k_{h}, \kappa_{m h}$ and $k_{v}$, $\kappa_{m v}$ respectively. The basic steady state is assumed to be quiescent and the temperature distributions are found to be

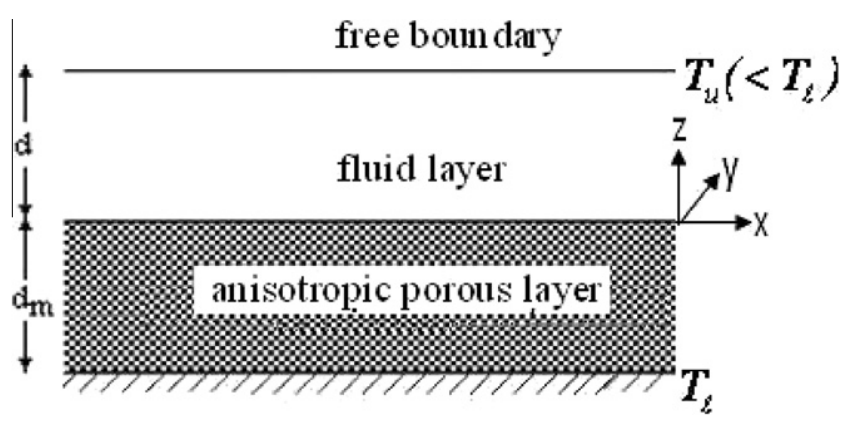

Fig. 1. Geometrical configuration of the system.
$T_{b}(z)=T_{0}-\frac{\left(T_{0}-T_{u}\right) z}{d} \quad 0 \leq z \leq d$

$T_{m b}\left(z_{m}\right)=T_{0}-\frac{\left(T_{\ell}-T_{0}\right) z_{m}}{d_{m}} \quad-d_{m} \leq z_{m} \leq 0$

where $T_{0}=\left(\kappa_{m v} T_{\ell} d+\kappa T_{u} d_{m}\right) /\left(\kappa_{m v} d+\kappa d_{m}\right)$ is the interface temperature and suffix $b$ denotes the basic state. Determination of the onset of convection is achieved by assuming that instability occurs by a way of a perturbation to the existing basic state in the form

$\vec{V}=\vec{V}^{\prime}, \quad T=T_{b}(z)+T^{\prime}, \quad p=p_{b}(z)+p^{\prime}, \quad \vec{V}_{m}=\vec{V}_{m}^{\prime}$,

$T_{m}=T_{m b}(z)+T_{m}^{\prime}, \quad p_{m}=p_{m b}(z)+p_{m}^{\prime}$

where the primed quantities are perturbations and assumed to be small. Equation (8) is substituted in Eqs. (1)-(6) and linearized in the usual manner. The pressure is eliminated from the momentum equations by operating $\nabla \times \nabla \times$ and only the vertical component is retained. The variables are then non-dimensionalized using $d, d^{2} / \kappa, \kappa / d$ and $T_{0}-T_{u}$ as the units of length, time, velocity, and temperature in the fluid layer and $d_{m}, d_{m}^{2} / \kappa_{m v}, \kappa_{m v} / d_{m}$ and $T_{\ell}-T_{0}$ as the corresponding characteristic quantities in the porous layer. It may be noted that separate length scales are chosen for the two layers so that each layer is of unit depth. In this manner, the detailed flow fields in both fluid and porous layers can be clearly discerned for all depth ratios, $\zeta=d / d_{m}$. The non-dimensional disturbance equations are then given by

$\left(\frac{1}{\operatorname{Pr}} \frac{\partial}{\partial t}-\nabla^{2}\right) \nabla^{2} w=0$

$\left(\frac{\partial}{\partial t}-\nabla^{2}\right) T=w$

$\left(\frac{D a}{P r_{m}} \frac{\partial}{\partial t}+\xi \nabla_{m h}^{2}+\frac{\partial^{2}}{\partial z_{m}^{2}}\right) w_{m}=0$

$\left(A \frac{\partial}{\partial t}-\frac{\partial^{2}}{\partial z_{m}^{2}}-\eta \nabla_{m h}^{2}\right) T_{m}=w_{m}$

where $D a=k_{v} / d_{m}^{2}$ is the Darcy number, $P r=v / \kappa$ is the Prandtl number, $\xi=k_{h} / k_{v}$ is the mechanical anisotropy parameter, $\eta=\kappa_{m h} / \kappa_{m v}$ is the thermal anisotropy parameter. From Eqs. (9) and (11), it is observed that the inertia has no influence on the stability criteria since the basic state whose stability is being analyzed is quiescent.

The boundary conditions are:

$w=\frac{\partial T}{\partial z}+B i T=0 \quad$ at $z=1$

$\frac{\partial^{2} w}{\partial z^{2}}=M \nabla_{h}^{2} T \quad$ at $z=1$

$w_{m}=\frac{\partial T_{m}}{\partial z_{m}}=0 \quad$ at $z=1$

Here, $B i=h d / \kappa$ is the Biot number and $M=\sigma_{T}\left(T_{0}-T_{u}\right) d / \mu \kappa$ is the Marangoni number, where $h$ is the heat transfer coefficient. At the interface (i.e. at $z=0$ ) the normal component of velocity, temperature, heat flux and the normal stress are continuous. Since there is no viscous stress term in the Darcy equation, continuity of shear stress across the interface cannot be used. Instead we use the experimentally suggested condition proposed by Beavers-Joseph [22], or its generalization due to Jones [23]. Accordingly, the following conditions at the interface are used: 
$w=\frac{\zeta}{\varepsilon_{T}} w_{m}$

$T=\frac{\varepsilon_{T}}{\zeta} T_{m}$

$\frac{\partial T}{\partial z}=\frac{\partial T_{m}}{\partial z_{m}}$

$\left(3 \nabla_{h}^{2}+\frac{\partial^{2}}{\partial z^{2}}\right) \frac{\partial w}{\partial z}=-\frac{\zeta^{4}}{\varepsilon_{T}}\left[\frac{1}{D a \xi}\right] \frac{\partial w_{m}}{\partial z_{m}}$

$\frac{\partial^{2} w}{\partial z^{2}}-\gamma \nabla_{h}^{2} w=\frac{\beta \zeta}{\sqrt{D a \xi}} \frac{\partial w}{\partial z}-\frac{\beta \zeta^{3}}{\varepsilon_{T}}\left[\frac{1}{\sqrt{D a \xi}}\right] \frac{\partial w_{m}}{\partial z_{m}}$

where $\varepsilon_{T}=\kappa / \kappa_{m v}$ is the ratio of thermal diffusivities, $\beta$ is the slip parameter, $\nabla_{h}^{2}=\partial^{2} / \partial x^{2}+\partial^{2} / \partial y^{2}$ is the horizontal Laplacian operator. The constant $\gamma$ takes the value 0 for the Beavers-Joseph condition and 1 for the Jones condition. Since the principle of exchange of instabilities holds for surface tension driven convection either in a fluid layer (see $[24,25]$ ) or in a porous layer $[4,5]$ heated form below, it is reasonable to assume that it holds good even for the present configuration as well. Further, the numerical calculations carried out for a wide range of parameters by Straughan [11] also corroborates the validity of principle of exchange of stability for the superposed system. Hence, the time derivatives will be dropped conveniently from Eqs. (11)-(14). Then performing a normal mode expansion of the dependent variables in both fluid and porous layers as

$(w, T)=[W(z), \Theta(z)] \exp [i(\ell x+m y)]$

$\left(w_{m}, T_{m}\right)=\left[W_{m}\left(z_{m}\right), \Theta_{m}\left(z_{m}\right)\right] \exp \left[i\left(\tilde{\ell} x_{m}+\tilde{m} y_{m}\right)\right]$

and substituting them in Eqs. (11)-(14) (with $\partial / \partial t=0)$, we obtain the following ordinary differential equations

$\left(D^{2}-a^{2}\right)^{2} W=0$

$\left(D^{2}-a^{2}\right) \Theta=-W$

$\left(D_{m}^{2}-\xi a_{m}^{2}\right) W_{m}=0$

$\left(D_{m}^{2}-\eta a_{m}^{2}\right) \Theta_{m}=-W_{m}$

where $D$ and $D_{m}$ denote differentiation with respect to $z$ and $z_{m}$ respectively, $a=\sqrt{\ell^{2}+m^{2}}$ and $a_{m}=\sqrt{\tilde{\ell}^{2}+\tilde{m}^{2}}$ are correspondingly the overall horizontal wave numbers in the fluid and porous layers. If matching of the solutions in the two layers is to be possible, the wave numbers must be same for the fluid and porous layers, so that we have $a / d=a_{m} / d_{m}$, and hence $\zeta=a / a_{m}$.Using Eqs. (21) and (22), the boundary conditions now become

$$
\begin{aligned}
& {\left[D^{2}-3 a^{2}\right] D W=-\frac{\zeta^{4}}{\varepsilon_{T} D a \xi} D_{m} W_{m}} \\
& {\left[D^{2}+\gamma a^{2}-\frac{\beta \zeta D}{\sqrt{D a \xi}}\right] W=-\frac{\beta \zeta^{3}}{\varepsilon_{T} \sqrt{D a \xi}} D_{m} W_{m}}
\end{aligned}
$$

Thus the problem has now been reduced to an eigenvalue problem consisting of a sixth order ordinary differential equation in the fluid layer and a fourth order ordinary differential equation in the porous layer subject to five boundary as well as five interface conditions.

\section{Method of solution}

The solution to the eigenvalue problem is obtained exactly. We note that Eqs. (23) and (25) are independent of $\Theta$ and $\Theta_{m}$ so that they can be solved for $W$ and $W_{m}$ to get

$W=A\left[\cosh (a z)+\Delta_{1} \sinh (a z)+\Delta_{2} z \cosh (a z)+\Delta_{3} z \sinh (a z)\right]$

$W_{m}=A \frac{\varepsilon_{T}}{\zeta}\left[\operatorname{coth}\left(a_{m} \sqrt{\xi}\right) \sinh \left(a_{m} \sqrt{\xi} z_{m}\right)+\cosh \left(a_{m} \sqrt{\xi} z_{m}\right)\right]$

where

$\Delta_{1}=\frac{\zeta^{3} a_{m} \sqrt{\xi} \operatorname{coth}\left(\sqrt{\xi} a_{m}\right)}{2 a^{3} D a \xi}$

$\Delta_{2}=-\frac{2 a\left(\cosh a+\Delta_{1} \sinh a\right)-\sinh a\left(a^{2}(\gamma+1)+\frac{\beta \zeta^{2} \operatorname{coth}\left(\sqrt{\xi} a_{m}\right)}{\sqrt{D a \xi}}-\frac{\beta \zeta a \Delta_{1}}{\sqrt{D a \xi}}\right)}{2 a \cosh a+\beta \zeta \sinh a / \sqrt{D a \xi}}$

$\Delta_{3}=-\left(1+\Delta_{2}\right) \operatorname{coth} a-\Delta_{1}$

Substituting for $W$ and $W_{m}$ thus obtained in Eqs. (24) and (26) respectively and solving we obtain $\Theta$ and $\Theta_{m}$ in the form

$$
\begin{aligned}
\Theta= & \frac{A}{4 a^{2}}\left[K_{1} \sinh (a z)+L_{1} \cosh (a z)+f(z)\right] \\
\Theta_{m}= & A\left[\left\{\frac{a K_{1}-\lambda_{4}}{\sqrt{\eta} a_{m}} \sinh \left(\sqrt{\eta} a_{m} z_{m}\right)-\frac{\varepsilon_{T} z_{m}}{2 \zeta \sqrt{\xi} a_{m}} \sinh \left(\sqrt{\xi} a_{m} z_{m}\right)\right\}\right. \\
& \left.+\left\{\frac{\varepsilon_{T} L_{1}}{\zeta} \cosh \left(\sqrt{\eta} a_{m} z_{m}\right)-\frac{\varepsilon_{T} \operatorname{coth}\left(\sqrt{\xi} a_{m} z_{m}\right)}{2 \zeta \sqrt{\xi} a_{m}} \cosh \left(\sqrt{\xi} a_{m} z_{m}\right)\right\}\right]
\end{aligned}
$$

Here,

$K_{1}=\left(\lambda_{1} \cosh a+\lambda_{2} \sinh a-L_{1}(a \sinh a+B i \cosh a)\right) /(a \cosh a+B i$ $\times \sinh a)$

$L_{1}=\frac{(a \cosh a+B i \sinh a)\left(\lambda_{3}-\lambda_{4} \cosh \left(\sqrt{\eta} a_{m}\right)\right)+\cosh \left(\sqrt{\eta} a_{m}\right)\left(\lambda_{1} \cosh a+\lambda_{2} \sinh a\right)}{\sqrt{\eta} a_{m} \zeta \sinh \left(\sqrt{\eta} a_{m}\right)(a \cosh a+B i \sinh a) / \varepsilon_{T}+a \cosh \left(\sqrt{\eta} a_{m}\right)(a \sinh a+B i \cosh a)}$

$W=D \Theta+B i \Theta=0 \quad$ at $z=1$

$D^{2} W+M a^{2} \Theta=0$ at $z=1$

$W_{m}=D_{m} \Theta_{m}=0 \quad$ at $z_{m}=-1$

and those at the interface (i.e. at $z=0$ ) are

$W=\frac{\zeta}{\varepsilon_{T}} W_{m}$

$D \Theta=D_{m} \Theta_{m}$

$\Theta=\frac{\varepsilon_{T}}{\zeta} \Theta_{m}$

$$
\begin{aligned}
f(z)= & {\left[\left(\Delta_{3}-2 a\right) z-\Delta_{2} a z^{2}\right] \sinh (a z) } \\
& +\left[\Delta_{3} a z^{2}+\left(\Delta_{2}-2 a \Delta_{1}\right) z\right] \cosh (a z)
\end{aligned}
$$

with

$$
\begin{aligned}
& \lambda_{1}=\frac{1}{4 a^{2}}\left[\left\{2 a^{2}+\left(a^{2}-1\right) \Delta_{2}+2 \Delta_{1} a+a \Delta_{3}\right\}+B i\left\{-\Delta_{2}+2 \Delta_{1} a+\Delta_{3} a\right\}\right] \\
& \lambda_{2}=\frac{1}{4 a^{2}}\left[\left\{2 a+2 a^{2} \Delta_{1}+a \Delta_{2}+\left(a^{2}-1\right) \Delta_{3}\right\}+B i\left\{2 a+\Delta_{2} a-\Delta_{3}\right\}\right]
\end{aligned}
$$




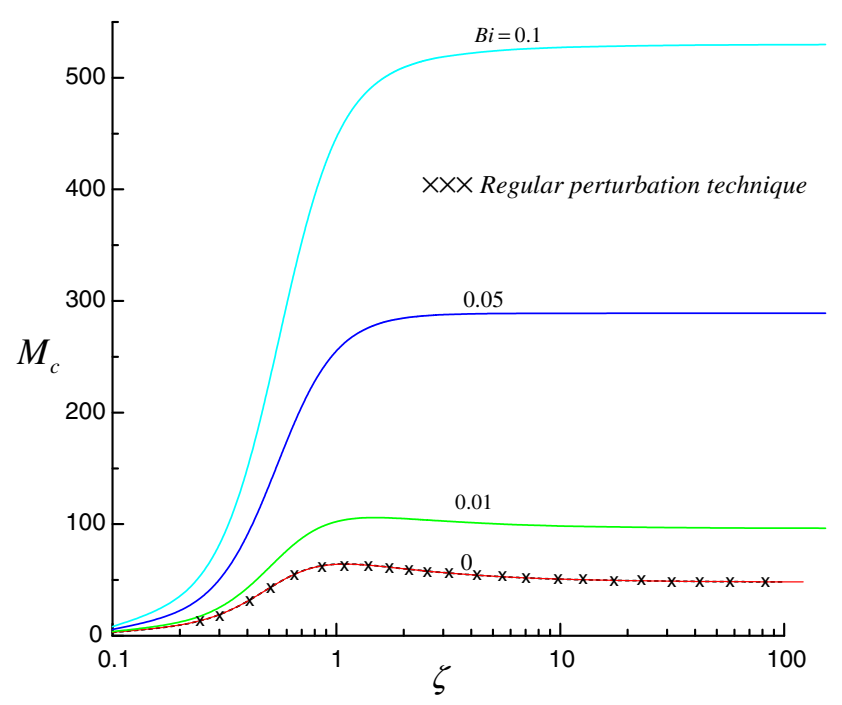

Fig. 2a. Variation of $M_{c}$ with $\zeta$ for different values of $B i$ when $\eta=0.5, \xi=0.5$, $\varepsilon_{T}=0.725, \beta=1$ and $D a=0.003$.

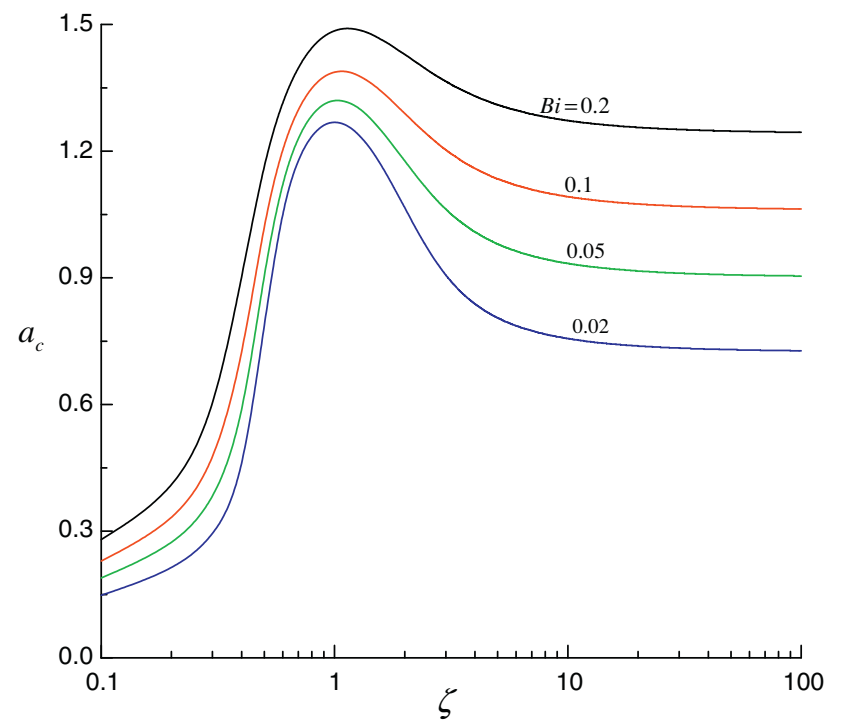

Fig. 2b. Variation of $a_{c}$ with $\zeta$ for different values of $B i$ when $\eta=0.5, \xi=0.5$, $\varepsilon_{T}=0.725, \beta=1, D a=0.003$.

$$
\begin{aligned}
\lambda_{3}= & \frac{\varepsilon_{T}}{2 \zeta \sqrt{\xi} a_{m}}\left\{\left(\sqrt{\xi} a_{m}-\operatorname{coth}\left(\sqrt{\xi} a_{m}\right) \cosh \left(\sqrt{\xi} a_{m}\right)\right.\right. \\
& \left.-\left(a_{m} \operatorname{coth}\left(\sqrt{\xi} a_{m}\right)-1\right) \sinh \left(\sqrt{\xi} a_{m}\right)\right\}
\end{aligned}
$$

$\lambda_{4}=\frac{1}{4 a^{2}}\left\{2 a \Delta_{1}-\Delta_{2}-2 a \Delta_{3}\right\}$

$\lambda_{5}=\frac{\varepsilon_{T}}{2 \zeta\left(\sqrt{\xi} a_{m}\right)}\left(\operatorname{coth}\left(\sqrt{\xi} a_{m}\right) \cosh \left(\sqrt{\xi} a_{m}\right)-\sinh \left(\sqrt{\xi} a_{m}\right)\right)$

The coupled boundary condition given by Eq. (28) is used ultimately to obtain an expression for the Marangoni number in the form

$M=\frac{4 a\left[\left(a+\Delta_{2} a+2 \Delta_{3}\right) \cosh a+\left(2 \Delta_{2}+\Delta_{1} a+\Delta_{3} a\right) \sinh a\right]}{K_{1} \sinh a+L_{1} \cosh a+f(1)}$

The critical Marangoni number $M_{c}$ is obtained numerically by minimizing $M$ with respect to the wave number $a$ for various values of

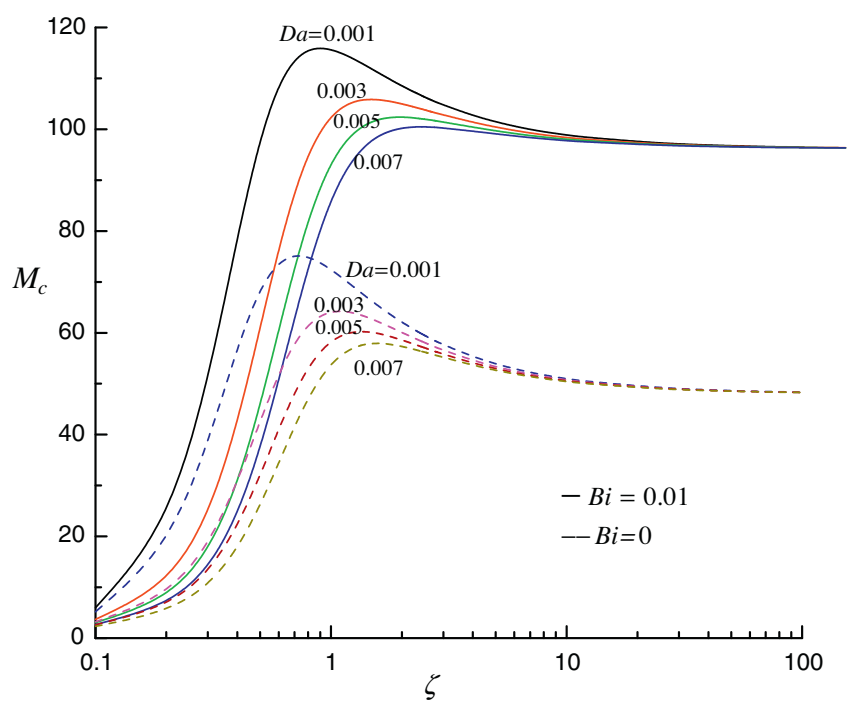

Fig. 3a. Variation of $M_{c}$ with $\zeta$ for different values of $D a$ when $\eta=0.5, \xi=0.5$, $\varepsilon_{T}=0.725, \beta=1$.

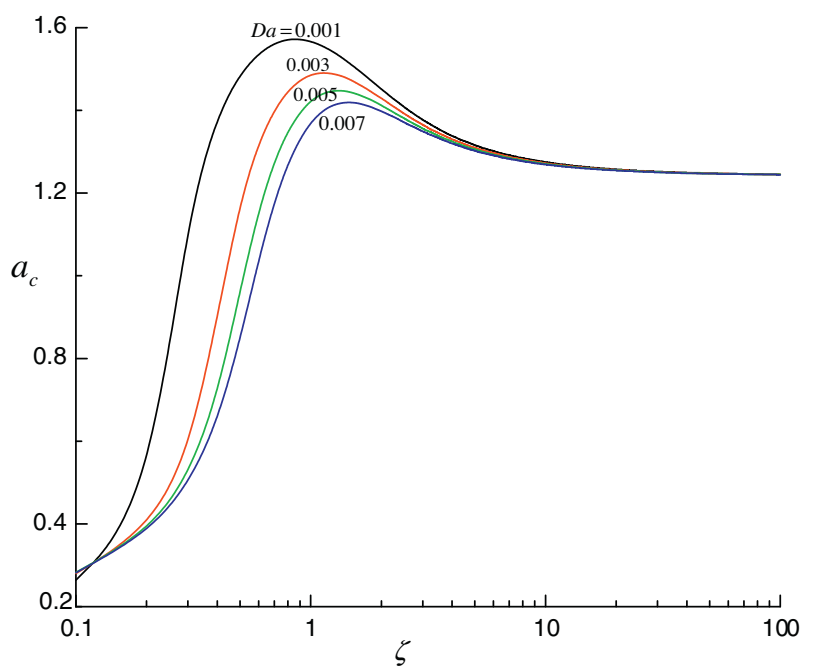

Fig. 3b. Variation of $a_{c}$ with $\zeta$ for different values of $D a$ when $\eta=0.5, \xi=0.5$, $\varepsilon_{T}=0.725, \beta=1, B i=0.2$.

$\zeta, \varepsilon_{T}, D a, \xi, \beta, B i$ and $\eta$ and the results are exhibited graphically in Figs. $2-5$ and also in Tables $1-5$.

\section{Results and discussion}

The onset of surface-tension-driven convection due to temperature gradients in a system consisting of a fluid layer overlying a fluid saturated anisotropic porous layer is studied. The eigenvalue problem is solved exactly and an analytical expression for the Marangoni number is obtained. When both boundaries are insulating to temperature perturbations (i.e., $D \Theta=0$ at $z=0,1$ ), the problem is also solved by regular perturbation technique with wave number $a$ as a perturbation parameter and an expression for the critical Marangoni number is obtained in the form (for details see [16])

$M_{c}=\frac{\left(\frac{\varepsilon_{T}}{\zeta}+\frac{\eta}{\zeta^{2}}\right)}{C_{1}+C_{2} / 2+C_{3} / 3+C_{4} / 4+C_{5} / 2 \zeta^{2}}$ 


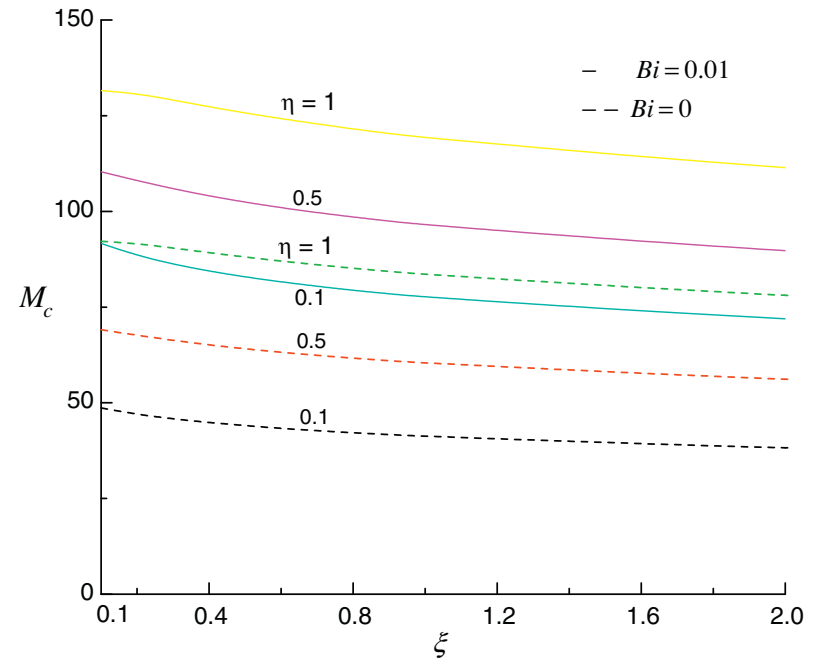

Fig. 4a. Variation of $M_{c}$ with $\xi$ for different values of $\eta$ when $\zeta=1, \varepsilon_{T}=0.725, \beta=1$, $D a=0.003$.

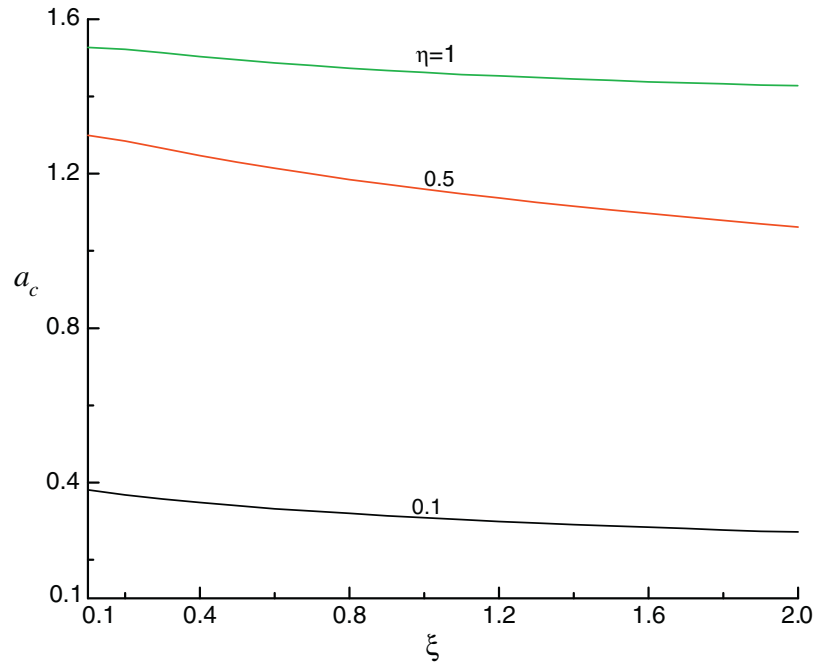

Fig. 4b. Variation of $a_{c}$ with $\xi$ for different values of $\eta$ when $\zeta=1, \varepsilon_{T}=0.725, \beta=1$, $D a=0.003, B i=0.001$

where

$C_{1}=\frac{\sqrt{D a \xi} \varepsilon_{T} / \beta \zeta^{2}+\varepsilon_{T} / 2 \zeta}{1+\zeta^{2} / \beta \sqrt{D a}+\zeta+\zeta^{3} / 3 D a \xi}$,

$C_{2}=\left(\frac{\zeta^{2}+\zeta}{\beta \sqrt{D a}}\right) C_{1}-\frac{\sqrt{D a \xi} \varepsilon_{T}}{\beta \zeta^{2}}, \quad C_{3}=\frac{\zeta^{2} C_{1}}{2 D a \xi}-\frac{\varepsilon_{T}}{2 \zeta}$

$C_{4}=-\frac{\zeta^{3} C_{1}}{6 D a \xi}, \quad C_{5}=C_{6}=C_{1} \zeta \varepsilon_{T}$

As mentioned earlier, at the interface of the fluid and anisotropic porous layers both the Beavers-Joseph and the Jones conditions are used to examine their influence on the criterion for the onset of Marangoni convection. The results obtained by employing these two boundary conditions for different values of $\zeta, \eta$ and $\beta$ for fixed values of other parameters are compared in Tables 1 and 2. From the tables, it is seen that the critical Marangoni numbers almost remain the same for these two types of boundary conditions and thus justifies the use of classical Beavers-Joseph slip condition in trickling flow situations. The numerically evaluated critical stability

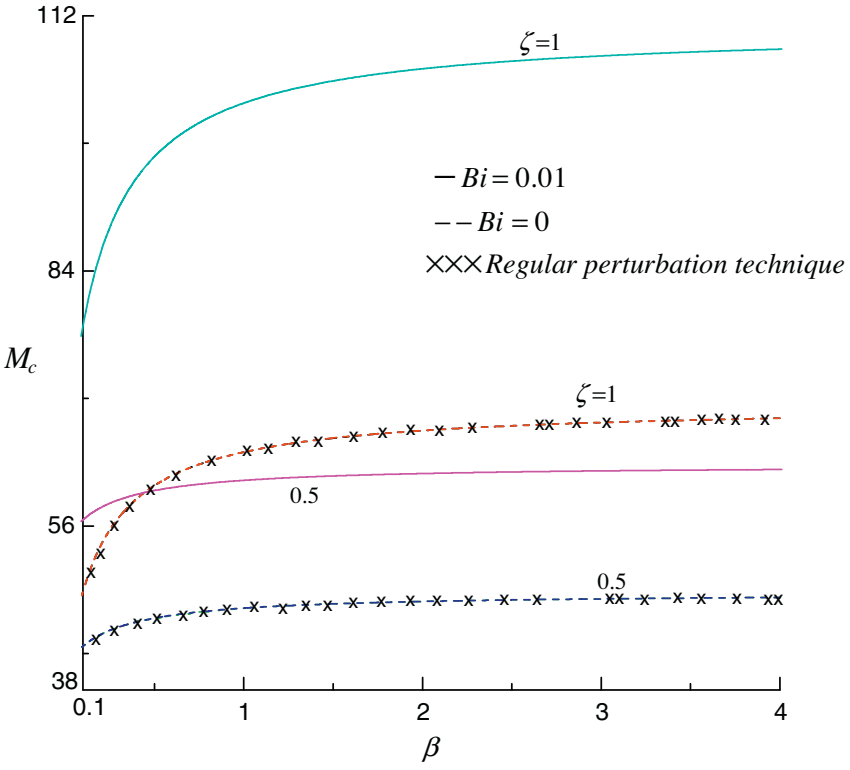

Fig. 5. Variation of $M_{c}$ with $\beta$ for two values of $\zeta$ and $B i$ when $\eta=0.5, \xi=0.5, \varepsilon_{T}=0.725, D a=0.003$.

Table 1

Comparison of critical Marangoni numbers for Beavers-Joseph (BJ) and Jones conditions for $B i=0.01, \xi=0.1, D a=0.003, \beta=1, \varepsilon_{T}=0.725$.

\begin{tabular}{|c|c|c|c|c|c|c|}
\hline \multirow[t]{2}{*}{$\zeta$} & \multicolumn{3}{|l|}{$\begin{array}{l}M_{C}(\mathrm{BJ}) \\
\eta\end{array}$} & \multicolumn{3}{|c|}{$\begin{array}{l}M_{c} \text { (Jones) } \\
\eta\end{array}$} \\
\hline & 0.1 & 0.5 & 1.0 & 0.1 & 0.5 & 1.0 \\
\hline 0.2 & 7.095 & 10.262 & 12.357 & 7.098 & 10.267 & 12.363 \\
\hline 0.4 & 31.824 & 42.033 & 50.721 & 31.823 & 42.033 & 50.722 \\
\hline 0.6 & 58.917 & 76.579 & 94.88 & 58.916 & 76.578 & 94.879 \\
\hline 0.8 & 74.811 & 94.982 & 118.035 & 74.811 & 94.981 & 118.035 \\
\hline 1.0 & 82.891 & 102.428 & 125.732 & 82.89 & 102.427 & 125.732 \\
\hline
\end{tabular}

Table 2

Comparison of critical Marangoni numbers for Beavers-Joseph (BJ) and Jones conditions for $\eta=0.5=\xi, D a=0.003, \zeta=1, \varepsilon_{T}=0.725$ and $B i=0.1$.

\begin{tabular}{lllllll}
\hline$\beta$ & 0.1 & 0.3 & 0.5 & 1 & 2 & 3 \\
\hline$M_{c}$ (BJ) & 340.408 & 397.03 & 421.533 & 447.211 & 463.633 & 469.808 \\
$M_{c}$ (Jones) & 340.403 & 397.025 & 421.530 & 447.209 & 463.632 & 469.807 \\
\hline
\end{tabular}

Table 3

Comparison of critical Marangoni number with those of Pearson [24] for a single fluid layer case.

\begin{tabular}{lllll}
\hline$B i$ & 0 & 0.01 & 0.05 & 0.1 \\
\hline$M_{c}$ (present study) & 48.024 & 96.412 & 289.091 & 529.842 \\
$M_{c}$ (Pearson [24]) & 48.02 & 96.346 & 289.07 & 529.82 \\
\hline
\end{tabular}

parameters for various values of physical parameters by utilizing the Beavers-Joseph slip condition are presented graphically in Figs. 2-5 and also tabulated in Tables 3-5.

Fig. 2a shows the plot of critical Marangoni number $M_{c}$ as a function of depth ratio $\zeta$ for different values of Biot number $B i$ when $\varepsilon_{T}=0.725, D a=0.003, \beta=1, \eta=0.5$ and $\xi=0.5$. Small values of $\zeta$ correspond to the single porous layer case, while large values of $\zeta$ correspond to the single fluid layer case. From Fig. 2a, it is seen that increasing $\mathrm{Bi}$ is to increase the critical Marangoni number and hence its effect is to delay the onset of Marangoni convection. This may be due to the fact that with increasing $B i$, the thermal disturbances easily dissipate into the ambient surrounding due to a 
Table 4

Critical Marangoni number for different values of anisotropic parameters and the Darcy number when $B i=0.01, \beta=1, \varepsilon_{T}=0.725, \zeta=1.0$ for (a) $\eta=0.1$ nd (b) $\xi=0.1$.

\begin{tabular}{|c|c|c|c|c|}
\hline \multirow[t]{2}{*}{$\xi$} & \multicolumn{4}{|l|}{$\begin{array}{l}M_{c} \\
D a\end{array}$} \\
\hline & 0.001 & 0.003 & 0.005 & 0.007 \\
\hline \multicolumn{5}{|l|}{$a$} \\
\hline 0.1 & 97.589 & 91.609 & 86.887 & 82.866 \\
\hline 0.2 & 95.886 & 88.488 & 82.897 & 78.283 \\
\hline 0.4 & 93.564 & 84.407 & 77.840 & 72.618 \\
\hline 0.6 & 91.877 & 81.582 & 74.450 & 68.914 \\
\hline 0.8 & 90.522 & 79.393 & 71.886 & 66.160 \\
\hline 1.0 & 89.377 & 77.600 & 69.822 & 63.973 \\
\hline \multirow[t]{3}{*}{$\eta$} & \multirow{2}{*}{\multicolumn{4}{|c|}{$\begin{array}{l}M_{c} \\
D a\end{array}$}} \\
\hline & & & & \\
\hline & 0.001 & 0.003 & 0.005 & 0.007 \\
\hline \multicolumn{5}{|l|}{$b$} \\
\hline 0.1 & 97.589 & 95.609 & 86.887 & 82.866 \\
\hline 0.2 & 103.465 & 96.466 & 90.946 & 86.273 \\
\hline 0.4 & 115.043 & 101.210 & 98.649 & 92.640 \\
\hline 0.6 & 126.396 & 105.845 & 105.847 & 98.475 \\
\hline 0.8 & 137.529 & 114.805 & 112.586 & 103.841 \\
\hline 1.0 & 148.451 & 123.373 & 118.910 & 108.794 \\
\hline
\end{tabular}

better convective heat transfer coefficient at the top free surface and hence makes the system more stable. Moreover, for a fixed value of $B i, M_{c}$ increases initially with $\zeta$, reaches a maximum and ultimately attains an asymptotic value with further increase in $\zeta$. The critical Marangoni numbers obtained by regular perturbation technique when $B i=0$ (i.e., when both boundaries are insulated to temperature perturbations) are also shown in Fig. 2a by marking $(x \times x)$ and it is observed that the results are in very good agreement with those obtained exactly. This suggests the applicability of regular perturbation technique in solving similar type of convection problems for which exact solution is not possible. From the figure we also note that, depending on the value of $B i, M_{c}$ approaches to an asymptotic value at higher values of $\zeta$ (i.e., single fluid layer case). The asymptotic values computed for different values of $B i$ are compared with those obtained from the expression given by Pearson [24] for a single fluid layer case and good agreement is found (see Table 3). Whereas, the curves of $M_{c}$ for different $B i$ come together as $\zeta \rightarrow 0$. The variation of critical wave number $a_{c}$ as a function $\zeta$ is shown in Fig. $2 \mathrm{~b}$ for the values presented in Fig. 2a. The figure indicates that increasing $B i$ is to increase the critical wave number (i.e., to decrease the size of convection cells) but remains almost invariant at smaller as well as at higher values of $\zeta$. The critical wave number is found to be negligibly small when $B i=0$ and for other values of $B i$ it takes the peak value around $\zeta=1$ (i.e., porous and fluid layers are of same depth).

The influence of permeability of the porous layer on the onset of Marangoni convection is exhibited in Fig. 3a when $\varepsilon_{T}=0.725$, $\beta=1, \eta=0.5$ and $\xi=0.5$ for two values of $B i(=0$ and 0.01 ). It is seen that decrease in $D a$ is to increase the critical Marangoni number and hence its effect is to delay the onset of Marangoni convection. For small values of $\zeta$ (i.e. a very thin fluid layer above the porous layer), $M_{c}$ takes small values as it is directly proportional to the fluid layer thickness $d$. At higher values of $\zeta$, however, the curves of $M_{c}$ for different $D a$ coalesce. Also, the curves of $B i=0$ lie below those of $B i=0.01$ indicating the insulating boundaries have less stabilizing effect on the system. Fig. $3 b$ represents the variation of $a_{c}$ for the values considered in Fig. 3b. Although increasing $D a$ is to decrease the critical wave number, a reverse trend could be seen at smaller values of $\zeta$. At higher values of $\zeta$, however, the curves of $a_{c}$ join together for different $D a$ and $a_{c}$ remains independent of $\zeta$.

The effect of mechanical and thermal anisotropy parameters on the onset of Marangoni convection is emphasized by depicting the variation of $M_{c}$ and $a_{c}$ over a range of mechanical anisotropy parameter $\xi$ for different values of thermal anisotropy parameter $\eta$ in Figs. 4a and $4 \mathrm{~b}$, respectively for a fixed value of $D a=0.003$, $\zeta=1, \beta=1$ and $\varepsilon_{T}=0.725$ with $B i=0$ and 0.01 . Besides, the critical Marangoni numbers computed for different values of $\xi, \eta$ and $D a$ for fixed values of $\beta=1, B i=0.01, \varepsilon_{T}=0.725$ and $\zeta=1.0$ are also tabulated in Table 4 . As seen from the figures, both $M_{c}$ and $a_{c}$ increase with the decrease in the value of $\xi$. This is because, decrease in $\xi$ corresponds to smaller horizontal permeability which in turn hinders the motion of the fluid in the horizontal direction. As a consequence, the conduction process in the porous medium becomes more stable and hence higher values of $M_{c}$ are needed for the onset of convection. The larger resistance to horizontal flow also leads to a shortening of the horizontal wavelength (i.e., increase in the horizontal wave number) at the onset of convection. The results presented in Fig. 4b corroborate this fact. In the same figures, it is also seen that for a fixed value of $\xi, M_{c}$ decreases with decreasing $\eta$. This may be attributed to the fact that, as $\eta$ decreases the horizontal thermal diffusivity also decreases. Thus heat cannot be transported through the porous layer and hence the horizontal temperature variations in the fluid required to sustain convection

Table 5

Critical Marangoni number for different values of depth ratio, Darcy number and Biot number when $\beta=1, \varepsilon_{T}=0.725, \xi=0.5=\eta$.

\begin{tabular}{|c|c|c|c|c|c|c|c|c|}
\hline \multirow[t]{2}{*}{$\zeta$} & \multicolumn{4}{|c|}{$\begin{array}{l}M_{c}(B i=0) \\
D a\end{array}$} & \multicolumn{4}{|c|}{$\begin{array}{l}M c(B i=0.01) \\
D a\end{array}$} \\
\hline & 0.001 & 0.003 & 0.005 & 0.007 & 0.001 & 0.003 & 0.005 & 0.007 \\
\hline 0.1 & 5.178 & 3.198 & 2.631 & 2.324 & 5.969 & 3.686 & 3.033 & 2.679 \\
\hline 0.5 & 68.934 & 42.717 & 31.999 & 26.042 & 98.456 & 61.012 & 45.703 & 37.195 \\
\hline 1.0 & 72.414 & 64.118 & 58.314 & 53.799 & 115.681 & 102.428 & 93.155 & 85.943 \\
\hline 1.5 & 66.136 & 62.651 & 60.069 & 57.917 & 111.763 & 105.874 & 101.511 & 97.874 \\
\hline 2.0 & 62.091 & 60.058 & 58.567 & 57.317 & 108.540 & 104.985 & 102.379 & 100.194 \\
\hline \multirow[t]{3}{*}{2.5} & 59.465 & 58.055 & 57.038 & 56.190 & 106.326 & 103.805 & 101.986 & 100.469 \\
\hline & \multicolumn{4}{|c|}{$\begin{array}{l}M_{C}(B i=0.05) \\
D a\end{array}$} & \multicolumn{4}{|c|}{$\begin{array}{l}M_{c}(B i=0.1) \\
D a\end{array}$} \\
\hline & 0.001 & 0.003 & 0.005 & 0.007 & 0.001 & 0.003 & 0.005 & 0.007 \\
\hline 0.1 & 9.133 & 5.640 & 4.641 & 4.100 & 13.087 & 8.082 & 6.650 & 5.875 \\
\hline 0.5 & 216.545 & 134.190 & 100.519 & 81.808 & 364.157 & 225.663 & 169.039 & 137.573 \\
\hline 1.0 & 288.745 & 255.664 & 232.521 & 214.517 & 505.075 & 447.211 & 406.728 & 375.236 \\
\hline 1.5 & 294.273 & 278.767 & 267.279 & 257.702 & 522.410 & 494.884 & 474.489 & 457.487 \\
\hline 2.0 & 294.336 & 284.695 & 277.629 & 271.704 & 526.580 & 509.332 & 496.690 & 486.090 \\
\hline 2.5 & 293.768 & 286.803 & 281.778 & 277.586 & 528.071 & 515.551 & 506.518 & 498.982 \\
\hline
\end{tabular}


are less efficiently dissipated for small $\eta$. Hence, the base state becomes less stable leading to lower values of $M_{c}$ and also the onset of Marangoni convection occurs at a higher wave number. The results presented for different values of $D a$ in Table 4 also confirm the above observations. Table 5 contains the values of $M_{c}$ for different values of $\zeta, D a$ and $B i$ when $\beta=1, \varepsilon_{T}=0.725, \xi=0.5=\eta$. It is noted that, depending on the values of $B i$ and $D a$, there is a threshold value of $\zeta \geqslant 1$ that produces maximum stability to the system. In fact, this threshold value increases with increasing $B i$ and $D a$.

Fig. 5 depicts the variation of $M_{c}$ as a function of slip parameter $\beta$ for two values of $\zeta(=0.5$ and 1$)$ and $B i(=0$ and 0.01$)$ with $\eta=0.5=\xi, \varepsilon_{T}=0.725$ and $D a=0.003$. It is observed that increase in the slip parameter is to increase the critical Marangoni number initially but remains almost invariant at higher values of the same. Moreover, the effect of slip parameter on the onset of Marangoni convection is found to be more pronounced with an increase in the value of $\zeta$.

\section{Conclusions}

The criterion for the onset of Marangoni convection in an anisotropic porous layer underlain by a fluid layer is investigated theoretically to understand control of Marangoni convection. The Darcy equation is used in the porous medium and both Beavers-Joseph and Jones conditions are used at the interface between the porous and fluid layers. The top of the fluid layer is free at which surface tension variations due to temperature are allowed for and a general thermal boundary condition is invoked. The bottom of the porous layer is rigid and considered to be insulating to temperature perturbations. The resulting eigenvalue problem is solved exactly and also by a regular perturbation technique when the top and bottom boundaries are insulating to temperature perturbations and it is found that the results complement with each other. The critical Marangoni and the corresponding wave numbers are determined over a wide range of values of the depth ratio $\zeta$, and degrees of anisotropy of the permeability $\xi$ and thermal diffusivity $\eta$, Biot number $B i$, Darcy number $D a$ and the slip parameter $\beta$. It is found that there is no preference between the Beavers-Joseph and Jones conditions since the critical stability parameters obtained from these two conditions are almost the same. It is observed that the mechanical and thermal anisotropy parameters influence the stability of the system considerably and there is a threshold value of $\zeta \geqslant 1$ that produces maximum stability to the system. Both $M_{c}$ and $a_{c}$ increase with decreasing $\xi$ and increasing $\eta$ but $a_{c}$ becomes vanishingly small when $B i=0$. Increasing $B i$ and decreasing $D a$ is to delay the onset of Marangoni convection and also to increase the critical wave number. The effect of increasing slip parameter is to delay the onset of Marangoni convection and its effect is found to be significant for depth ratios $\zeta \geqslant 0.5$. From the scenario envisaged, it is evident that it is possible to control Marangoni convection effectively in a composite fluid and anisotropic porous layers system by tuning the values of physical parameters involved therein namely, $\zeta, \beta, D a, \xi, B i$ and $\eta$.

\section{Acknowledgements}

One of the authors (ISS) wishes to thank the BK21 Program of the School of Mechanical Engineering, Yonsei University, Seoul, Korea for inviting him as a visiting Professor and also the Bangalore University for sanctioning sabbatical leave. Also, one of us (KBC) wishes to thank the Principal for his encouragement and support. We thank the reviewer for useful comments.

\section{References}

[1] K. Vafai (Ed.), Hand Book of Porous Media, Marcel Dekker, New York, 2000.

[2] K. Vafai (Ed.), Handbook of Porous Media, Taylor and Francis, CRC, Boca Raton, 2005.

[3] D.A. Nield, A. Bejan, Convection in Porous Media, second ed., Springer-Verlag, New York, 2006.

[4] M. Hennenberg, M.Z. Saghir, A. Rednikov, J.C. Legros, Porous media and the Benard-Marangoni problem, Transport Porous Med. 27 (1997) 327-355.

[5] N. Rudraiah, V. Prasad, Effect of Brinkman boundary layer on the onset of Marangoni convection in a fluid saturated porous layer, Acta Mech. 127 (1998) 235-246.

[6] I.S. Shivakumara, C.E. Nanjundappa, K.B. Chavaraddi, Darcy-BenardMarangoni convection in porous media, Int. J. Heat Mass Transfer 52 (2009) 2815-2823.

[7] D.A. Nield, Onset of convection in a fluid layer overlying a layer of a porous medium, J. Fluid Mech. 81 (1977) 513-522.

[8] M.E. Taslim, V. Narusawa, Thermal stability of horizontally superposed porous and fluid layers, ASME J. Heat Transfer 111 (1989) 357-362.

[9] F. Chen, Throughflow effects on convective instability in superposed fluid and porous layers, J. Fluid Mech. 23 (1990) 113-133.

[10] G. McKay, Onset of buoyancy-driven convection in superposed reacting fluid and porous layers, J. Eng. Math. 33 (1998) 31-46.

[11] B. Straughan, Surface-tension-driven convection in a fluid overlying a porous layer, Comput. Phys. 170 (2001) 320-337.

[12] B. Straughan, Effect of property variation and modelling on convection in a fluid overlying a porous layer, J. Numer. Anal. Methods Geomech. 26 (2002) 75-97.

[13] D.A. Nield, Modelling the effect of surface tension on the onset of natural convection in a saturated porous medium, Transport Porous Med. 31 (1998) 365-368.

[14] A. Khalili, I.S. Shivakumara, S.P. Suma, Convective instability in superposed fluid and porous layers with vertical throughflow, Transport Porous Med. 51 (2001) 1-18.

[15] M. Carr, Penetrative convection in a superposed porous-medium-fluid layer via internal heating, J. Fluid Mech. 509 (2004) 305-329.

[16] I.S. Shivakumara, S.P. Suma, K.B. Chavaraddi, Onset of surface-tensiondriven convection in superposed fluid and porous layers, Arch. Mech. 55 (2006) 327-348.

[17] I.S. Shivakumara, K.B. Chavaraddi, Marangoni convection in a composite porous layer and a fluid layer with a deformable free surface, Int. J. Fluid. Mech. Res. 34 (4) (2007) 352-373.

[18] Z. Alloui, P. Vasseur, Convection in superposed fluid and porous layers, Acta Mech. (2010), doi:10.1007/s00707-010-0284.

[19] H.E. Huppert, M.G. Worster, Binary solidification of a binary melt, Nature 314 (1985) 703-707.

[20] G. Castinel, M. Combarnous, Natural convection in an anisotropic porous layer, Rev. Gen. Therm. 14 (1975) 937-947.

[21] F. Chen, C.F. Chen, A.J. Pearlstein, Convective instability in superposed fluid and anisotropic porous layers, Phys. Fluids A 3 (4) (1991) 556-565.

[22] G.S. Beavers, D.D. Joseph, Boundary conditions at a naturally permeable wall, J. Fluid Mech. 30 (1967) 197-207.

[23] I.P. Jones, Low Reynolds number flow past a porous spherical shell, Proc. Camb. Philos. Soc. 73 (1973) 231-238

[24] J.R.A. Pearson, On convection cell induced by surface tension, J. Fluid Mech. 4 (1958) 489-500.

[25] A. Vidal, A. Acrivos, Nature of the neutral state in surface tension driven convection, Phys. Fluids 9 (1966) 615-616. 